\title{
Remote sensing comprehensive evaluation of land degradation based on relative rain use efficiency of vegetation
}

\author{
Yu Xing ${ }^{*}$, Jinzhong Yang, Yaqing Sun, Cunhao Jiang, and Dong Chen \\ China Aero Geophysical Survey and Remote Sensing Center for Natural Resources, 100083 Beijing, \\ China
}

\begin{abstract}
China is one of the countries with serious land degradation problems in the world. Land degradation directly threatens the food security of our country and determines the sustainable development of social economy. In this paper, the potential evapotranspiration and moisture index are calculated to divide the climatic zone of China. Based on the NPP and precipitation data, the national rRUE is obtained by the edge function. With the climate "relative benchmark", the rules of threshold analysis is used to comprehensively evaluate the land degradation in China. The results show that: excellent land is concentrated in northeast China, western Xinjiang and eastern Qinghai-Tibet Plateau; mildly degraded land is widely and sporadically distributed in the eastern plain of China; moderately degraded land and severely degraded land are concentrated in western Inner Mongolia, southern Xinjiang and northern Qinghai-Xizang Plateau.
\end{abstract}

\section{Forward}

Under the strong interference of human activities, land resources are faced with land degradation problems such as desertification, soil erosion, soil pollution, grassland degradation and sharp reduction of wetlands. The qualitative change process of land degradation is the transformation process from land resource types with high productivity or ecological service function to sandy land, bare land, bare rock gravel land, saline land and non-agricultural construction land which are difficult to use or have very low productivity[1]. From an ecological point of view, land degradation is the deterioration of plant growth conditions and the decline of land productivity. There is a very close relationship between vegetation cover and land degradation. Deforestation, overgrazing and vegetation destruction are the direct causes of land degradation. The study shows that there is a negative exponential relationship between vegetation cover and land degradation, and the correlation coefficient is 0.8 [2]. Vegetation degradation is not only the cause of land degradation, but also the result of land degradation. Rain use efficiency of vegetation (RUE) is a comprehensive index to describe the productivity and health status of vegetation in ecosystem. Yin selected the RUE with high time resolution from remote sensing inversion

*Corresponding author:63216429@qq.com 
as the evaluation index, and used the time series analysis method to evaluate the development trend of desertification in Inner Mongolia Autonomous region[3]. Wang monitored and evaluated the land degradation in Beijing-Tianjin wind-blown sand source area based on net primary productivity(NPP) and RUE[4]. Gao also discussed the remote sensing evaluation method of desertification based on RUE[5]. However, there are few studies on large-scale evaluation of land degradation. Based on NPP and climatic data, this paper selects the index of relative rain use efficiency of vegetation (rRUE), combined with the significance test of the correlation coefficient of NPP with time, quantitatively evaluates and rapidly monitors the situation of land degradation in China.

\section{Methods}

\subsection{Technical route}

First of all, the RUE is calculated from the NPP and precipitation data. Secondly, the potential evapotranspiration and moisture index are calculated by using meteorological data[6-7]. And then, with reference to relevant standards, the land of China is divided into 18 climate gradient zones. Next, based on the climate gradient zone, the edge function model is established, from which the relative rain use efficiency of vegetation is obtained, and the threshold analysis method is used to classify the land production capacity of rRUE. Finally, on the basis of considering the "relative benchmark" of climate, the significance of the correlation coefficient of NPP with time change is used. The annual average and annual maximum of rRUE are used to comprehensively evaluate the land degradation situation in China in the past 10 years. The research route is shown in Fig. 1.

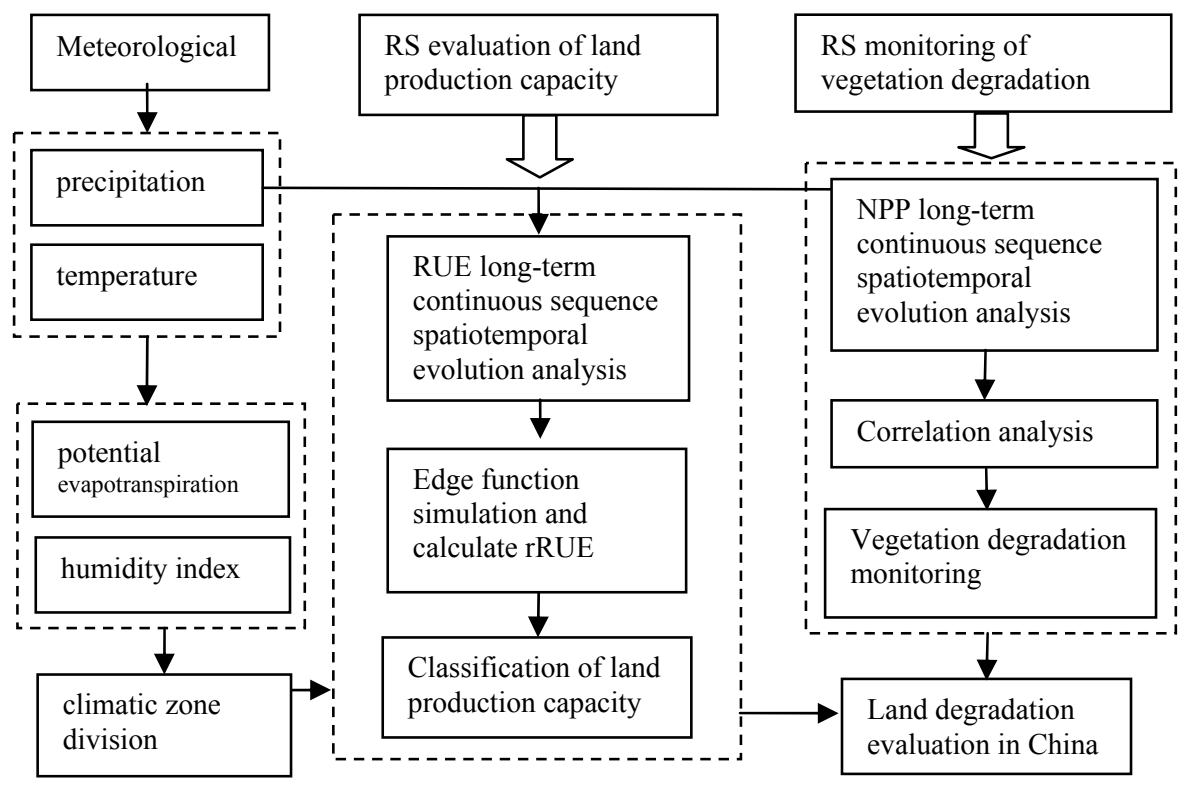

Fig.1. Technical route.

\subsection{Comprehensive evaluation}

The mean value of RUE (RUEmean) represents the long-term vegetation productivity and production capacity of the ecosystem, while maximum value of RUE (RUEmax) represents 
the resilience of the ecosystem after disturbance, especially the interference of the interannual variation of annual precipitation [4]. The threshold analysis is used to classify the land production capacity based on the mean value of rRUE (rRUEmean) and the maximum value of rRUE (rRUEmax). The evaluation method is as follows:

- Severely degraded land: when $r R U E$ mean $<0$ and $r R U E \max <0$ in the study area, it means that the ecosystem production capacity in this area is lower than the minimum of its potential production capacity, that is, the land in this area has almost no production capacity, and the resilience of ecosystem anti-interference is also lower than the minimum of its potential elasticity, that is, the land in this area is almost unable to restore its productivity and does not have ecological recovery elasticity. These areas are defined as extremely poor production capacity in the study.

- Moderately degraded land: when $r R U E$ mean $<0$ and $0 \leq r R U E \max \leq 1$ in the study area, it means that the ecosystem production capacity in this area is lower than the minimum of its potential production capacity, that is, the land production capacity in this area is very low, but the regional ecosystem has normal anti-interference resilience, and the land in this area can also restore its productivity. When $0 \leq r R U E$ mean $\leq 1$ and $r R U E \max <$ 0 in the study area, it means that the ecosystem production capacity in this area is within the normal potential range, and the land production capacity in this area is at the annual average level. But the resilience of ecosystem anti-interference in this area is lower than its minimum potential elasticity, that is, the anti-interference ability of land in this area is low and does not have ecological restoration elasticity.

- Mildly degraded land: when $0 \leq r R U E$ mean $\leq 1$ and $0 \leq r R U E \max \leq 1$ and $R$ npp $<-$ 0.5214 in the study area, it means that the ecosystem production capacity in these areas is within the normal potential range, the land production capacity in this area is at the annual average level, and the ecosystem has normal anti-interference recovery elasticity, the land in this area has the potential to restore productivity. However, $R \mathrm{npp}<-0.5214$ indicates that the vegetation is degraded. So, these areas are defined as land with mildly degraded land. ( $R$ npp represents the correlation coefficient of NPP index and wetness index with time, and 0.5214 is the critical value of significance test of correlation coefficient at 0.1 confidence level. )

- Good land: when $0 \leq r R U E$ mean $\leq 1$ and $0 \leq r R U E \max \leq 1$ and $R \mathrm{npp} \geq-0.5214$ in the study area, it means that the ecosystem production capacity in these areas is within the normal potential range, the land production capacity in this area is at the annual average level, and the ecosystem has normal anti-interference recovery elasticity, the land in this area has the potential to restore productivity. And $R \mathrm{npp} \geq-0.5214$ indicates that the vegetation is restored. So, these areas are defined as land with Good land.

- Excellent land: when $r R U E$ mean $>1$ and $0 \leq r R U E \max \leq 1$ in the study area, it means that the ecosystem production capacity of these areas is very strong, and the ecosystem has normal anti-interference resilience, and the land in this area has the potential to restore productivity. When $0 \leq r R U E$ mean $\leq 1$ and $r R U E \max >1$ in the study area, it means that the ecosystem production capacity in these areas is within the normal potential range, the land production capacity in this area is at the annual average level, and the ecosystem has a strong anti-interference resilience. When $r R U E$ mean $>1$ and $r R U E \max >1$ in the study area, it means that the ecosystem production capacity of these areas is very strong, and the ecosystem has a strong anti-interference recovery elasticity. This area is defined as land with excellent production capacity.

- Abnormal: when the study area $(r R U E$ mean $<0$ and $r R U E$ max $>1)$ or $(r R U E$ mean $>1$ and $r R U E \max <0$ ), it means that one index of rRUEmean and rRUEmax is less than 0 , while the other index is greater than 1 . Thus, it is defined as an anomaly area.

According to the above threshold analysis, combined with the correlation between NPP and $\mathrm{MI}$ and time, the rules of comprehensive evaluation of land degradation are 
summarized, as shown in Table 1. Rnpp represents the correlation coefficient of NPP index and wetness index with time, and 0.5214 is the critical value of significance test of correlation coefficient at 0.1 confidence level.

\section{Results}

The annual average temperature, precipitation, potential evapotranspiration, NPP and RUE data are obtained or calculated, and the edge function fitting equations of RUEmean and RUEmax are established. The results are shown in Table 1.

Table 1. RUE upper and RUE lower bound edge function models based on MI data.

\begin{tabular}{|c|c|l|}
\hline \multicolumn{1}{|c|}{$\boldsymbol{y}$} & $\boldsymbol{x}$ & \multicolumn{1}{c|}{ Edge function model } \\
\hline RUEmean & \multicolumn{1}{c|}{\begin{tabular}{c}
\multicolumn{1}{c|}{ M.05 } \\
MImean
\end{tabular}} & $\begin{array}{l}y=0.0582 x^{4}-0.3505 x^{3}+0.9242 x^{2}-1.1621 x+ \\
0.5539\end{array}$ \\
\hline RUEmean 0.95 & MImean & $\begin{array}{l}y=-1.3533 x^{6}+12.621 x^{5}-46.595 x^{4}+86.198 x^{3}- \\
81.714 x^{2}+32.773 x+2.3314\end{array}$ \\
\hline RUEmax & MImax & $\begin{array}{l}y=-0.0313 x^{6}+0.1013 x^{5}+0.304 x^{4}-1.5361 x^{3}+ \\
2.0817 x^{2}-1.2363 x+0.3768\end{array}$ \\
\hline RUEmax & MImax & $\begin{array}{l}y=-0.0343 x^{6}+1.3229 x^{5}-10.011 x^{4}+31.39 x^{3}- \\
45.935 x^{2}+27.314 x+1.1375\end{array}$ \\
\hline
\end{tabular}

The annual rRUEmean and annual rRUEmax are calculated according to the formula:

$$
r R U E=\left(R U E-R U E_{0.05}\right) /\left(R U E_{0.95}-R U E_{0.05}\right) .
$$

According to the rules of comprehensive evaluation of land degradation, the evaluation results of land degradation in China are obtained (Fig. 2).

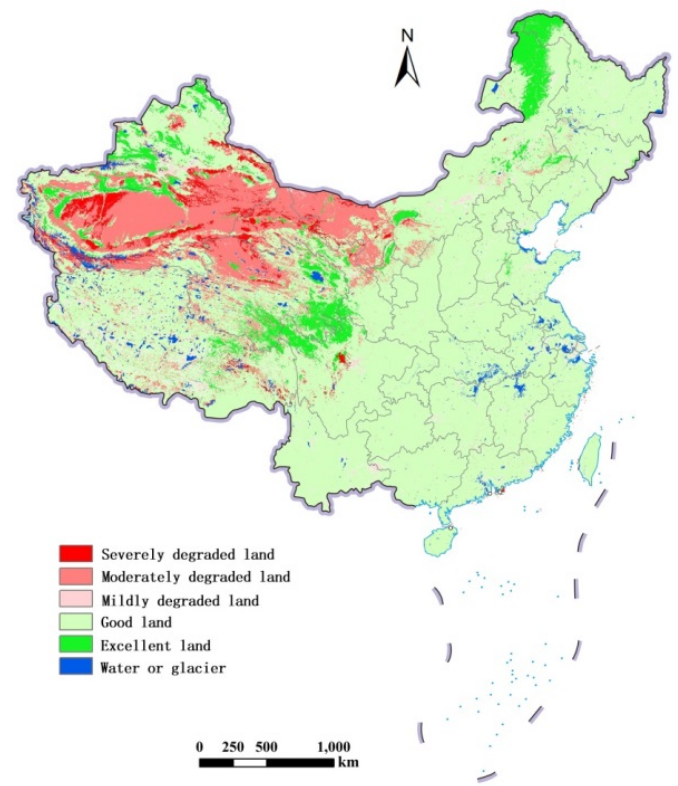

Fig. 2. Comprehensive evaluation of land degradation based on rRUE index by remote sensing.

The total area of degraded land in China is about $800000 \mathrm{~km}^{2}$, of which the areas of mild degradation, moderate degradation and severe degradation are about $460000 \mathrm{~km}^{2}$, 
$140000 \mathrm{~km}^{2}$ and $200000 \mathrm{~km}^{2}$ respectively, excellent land about $530000 \mathrm{~km}^{2}$ and good land about 8.17 million $\mathrm{km}^{2}$.

\section{Conclusion}

Based on the analysis of national regional climate and NPP data in recent 10 years, a remote sensing comprehensive evaluation map of land degradation in China is obtained based on NPP change trend and rRUE index. The study shows that excellent land is concentrated in northeast China, western Xinjiang and eastern Qinghai-Tibet Plateau; mildly degraded land is widely and sporadically distributed in the eastern plain of China; moderately degraded land and severely degraded land are concentrated in western Inner Mongolia, southern Xinjiang and northern Qinghai-Xizang Plateau.

\section{Acknowledgements}

This paper is supported by Programs of China Geological Survey (Remote sensing monitoring of national mining exploration and ecological spatial in key areas).

\section{References}

1. K.F. Zhang, X.W. Li, D.X. Zhang, J. F. Peng, J. Chen, Z. R. Yu, Environmental Science, 27,1244 (2006)

2. G.N. Sun, M.H. Wang, Journal of Arid Land Resources and Environment, 22,140 (2008)

3. H. Yin, Z.G. Li, Y.L. Wang, F. Cai, Acta Geographica Sinica,66,653 (2011)

4. H.Y. Wang. Assessment and monitoring of land degradation using NPP and vegetation rain use efficiency,Chinese Academy of Forestry (2013)

5. Z.H. Gao, Z.Y. Li, G.D. Ding, L. Y. Li, Science of Soil and Water Conservation, 3, 37 (2005)

6. Thornthwaite, Geographical Review, 38,55(1948)

7. S.W. Zhuang, H.C. Zuo, P.C. Ren, G. J. Xiong, B. D. Li, W. C. Dong, L. Y. Wang, Climatic and Environmental Research, 18,617(2013) 